\title{
Detection and significance of human papillomavirus, CDKN2A(p16) and CDKN1A(p21) expression in squamous cell carcinoma of the larynx
}

\author{
Rebecca D Chernock ${ }^{1,2}$, Xiaowei Wang ${ }^{3}$, Ge Gao ${ }^{3}$, James S Lewis Jr ${ }^{1,2}$, Qin Zhang ${ }^{4}$, \\ Wade L Thorstad $^{3}$ and Samir K El-Mofty ${ }^{1}$ \\ ${ }^{1}$ Department of Pathology and Immunology, Washington University School of Medicine, St Louis, MO, \\ USA; ${ }^{2}$ Department of Otolaryngology Head and Neck Surgery, Washington University School of Medicine, \\ St Louis, MO, USA; ${ }^{3}$ Department of Radiation Oncology, Washington University School of Medicine, \\ St Louis, MO, USA and ${ }^{4}$ Department of Biostatistics, Washington University School of Medicine, \\ St Louis, MO, USA
}

\begin{abstract}
Although a strong etiologic relationship between human papillomavirus (HPV) and a majority of oropharyngeal squamous cell carcinomas has been established, the role of HPV in non-oropharyngeal head and neck carcinomas is much less clear. Here, we investigated the prevalence and clinicopathologic significance of HPV and its reported biomarkers, CDKN2A(p16) and CDKN1A(p21), in laryngeal squamous cell carcinomas in patients treated either with primary surgery and postoperative radiation or with definitive radiation-based therapy. Nearly all of 76 tumors were keratinizing and none displayed the nonkeratinizing morphology that is typically associated with HPV infection in the oropharynx. However, CDKN2A(p16) immunohistochemistry was positive in 21 cases (28\%) and CDKN1A(p21) in 34 (45\%). CDKN2A(p16) and CDKN1A(p21) status strongly correlated with each other $(P=0.0038)$. Yet, only four cases were HPV positive by DNA in situ hybridization or by reverse transcriptase PCR E6/E7 mRNA (all four were CDKN2A(p16) and CDKN1A(p21) positive). Unexpectedly, 9 additional tumors out of 20 CDKN2A(p16) positive cases harbored high-risk HPV DNA by PCR. For further investigation of this unexpected result, in situ hybridization for E6/E7 mRNA was performed on these nine cases and all were negative, confirming the absence of transcriptionally active virus. Patients with CDKN1A(p21)-positive tumors did have better overall survival $(69 \%$ at 3 years) than those with CDKN1A(p21)-negative tumors (51\% at 3 years) $(P=0.045)$. There was also a strong trend towards better overall survival in the CDKN2A(p16)-positive group $(P=0.058)$. Thus, it appears that the role of HPV is more complex in the larynx than in the oropharynx, and that CDKN2A(p16) and CDKN1A(p21) expression may not reflect HPV-driven tumors in most cases. Because of this, CDKN2A(p16) should not be used as a definitive surrogate marker of HPV-driven tumors in the larynx. Modern Pathology (2013) 26, 223-231; doi:10.1038/modpathol.2012.159; published online 21 September 2012
\end{abstract}

Keywords: CDKN2A(p16); CDKN1A(p21); human papillomavirus; in situ hybridization; larynx; PCR

Human papillomavirus (HPV) can be detected in squamous cell carcinomas from various head and neck subsites. The highest rates of HPV infection are in the oropharynx with lower, yet still significant, rates of detection at other sites. Meta-analyses have

Correspondence: Dr SK El-Mofty, DMD, PhD, Department of Pathology and Immunology, Washington University School of Medicine, 660S. Euclid Avenue, Campus Box 8118, St Louis, MO 63110, USA.

E-mail: elmofty@path.wustl.edu

Received 26 June 2012; revised 17 July 2012; accepted 18 July 2012; published online 21 September 2012 reported HPV prevalence of $51 \%$ in tonsillar carcinomas and $20-25 \%$ for laryngeal or oral cavity carcinomas. ${ }^{1,2}$ However, the significance of finding the virus in a particular tumor is not always clear. The mere presence of HPV DNA in a tumor does not necessarily indicate that the virus is driving or contributing to tumor development or progression. Viral DNA may well be a 'bystander' as HPV DNA can be detected in non-tumor and even normal tissue from the upper aerodigestive tract. ${ }^{3,4}$ Detection methods that reflect transcriptionally active HPV, including CDKN2A(p16) immunohistochemistry and reverse transcriptase PCR (RT-PCR) or 
in situ hybridization for HPV DNA or E6/E7 mRNA, are, thus, necessary to demonstrate biologically significant virus. However, these methods have not been uniformly employed or are just emerging as viable tests out of formalin-fixed paraffin-embedded tissue.

The etiologic link between HPV and tumor development is by far the strongest for squamous cell carcinomas arising in the oropharynx, particularly in the tonsils and base of tongue. Here, biologically active HPV has been detected in a large number of tumors and is associated with unique clinical and pathologic features. HPV-positive tumors tend to occur in younger patients, are less strongly linked to tobacco and alcohol, and are associated with better patient outcomes compared with HPV-negative tumors. ${ }^{5,6}$ HPV-positive oropharyngeal squamous cell carcinomas can also be recognized histologically as the majority display nonkeratinizing morphology. ${ }^{7,8}$

At a molecular level, HPV-positive squamous cell carcinoma less frequently harbors gross chromosomal deletions than HPV-negative squamous cell carcinoma, and has a unique molecular signature characterized by overexpression of the tumor suppressor protein CDKN2A(p16) and infrequent TP53 mutations or EGFR amplification. ${ }^{9-11}$ Of these molecular markers, CDKN2A(p16) overexpression is of particular importance as it has emerged as robust surrogate biomarker of HPV-related oropharyngeal squamous cell carcinoma, where its overexpression reflects the presence of transcriptionally active HPV. ${ }^{12,13}$ Not surprisingly, CDKN2A (p16) overexpression is also observed in HPV-related cervical carcinomas. ${ }^{14}$ CDKN2A(p16) positivity approaches $100 \%$ in HPV-related oropharyngeal squamous cell carcinoma in some studies and in myriad other studies on oropharyngeal squamous cell carcinoma is positive in $85-95 \% .{ }^{9,12}$ In contrast, CDKN2A(p16) is usually consistently inactivated in HPV-negative head and neck cancers, leading to minimal, if any, detectable protein by immunohistochemistry. ${ }^{9,12}$

More recently, Hafkamp et al ${ }^{15}$ described overexpression of CDKN1A(p21), a tumor suppressor protein in the TP53 cascade, as another potential biomarker of HPV in tonsillar squamous cell carcinoma and a strong indicator of good prognosis. However, the association of CDKN1A(p21) expression with HPV and good prognosis in tonsillar squamous cell carcinoma has not been a universal finding. ${ }^{16,17}$

As the relationship between HPV and squamous cell carcinoma of the oropharynx unfolds, the significance of HPV in non-oropharyngeal head and neck squamous cell carcinoma remains less certain. In the larynx, HPV infection clearly occurs and is associated with human disease. Vertical transmission of HPV from mother to infant is thought to be the etiology of juvenile laryngeal papillomatosis. Patients with this disease develop multiple benign papillomas of the respiratory tract that have a particular predilection for the true vocal cord. Juvenile laryngeal papillomatosis is predominately caused by low-risk HPV subtypes 6 and $11 .^{18}$ Although malignant progression is rare, it appears to be more common in those papillomas harboring high-risk (HR) HPV subtypes, although it may be seen with low-risk HPV subtype infection as well. ${ }^{19}$

Although HPV DNA has also been detected in approximately one quarter of laryngeal squamous cell carcinomas on meta-analysis, the virus has been found in up to $19 \%$ of normal laryngeal mucosa samples as well, raising doubts as to its biological significance in tumor development or progression. ${ }^{20}$ Evaluation of the biological activity of the virus, either by assessment of HPV E6/E7 mRNA, DNA ISH or by utilizing surrogate biomarkers such as CDKN2A(p16), is important in determining whether or not HPV that is found in a tumor is actually having a role in tumor development or progression. However, these types of studies are few in number. ${ }^{21-24}$ Another factor casting doubt on the significance of HPV in laryngeal squamous cell carcinomas is the lack of consistency in the association of HPV with better patient survival. In fact, some studies of laryngeal squamous cell carcinoma have actually found decreased survival in patients with HPV DNA-positive tumors. ${ }^{25}$ This is in contrast to oropharyngeal squamous cell carcinoma where HPV has been shown to be strongly associated with favorable outcomes in virtually every study. ${ }^{5}$

The aim of this study was to investigate the frequency and transcriptional activity of HPV in squamous cell carcinoma of the larynx, and to assess its biological and clinical significance. As nonkeratinizing morphology, CDKN2A(p16) and CDKN1A (p21) overexpression, and better patient outcome are characteristic clinicopathologic features of transcriptionally active, HPV-related squamous cell carcinoma of the oropharynx, we assessed these features in laryngeal squamous cell carcinoma as well.

\section{Materials and methods}

\section{Case Selection}

The study was approved by the Human Research Protection Office of Washington University. Cases of laryngeal squamous cell carcinoma were identified from a large radiation oncology database maintained by one of the co-authors (WLT), which consisted of all patients with head and neck squamous cell carcinoma who received radiation therapy at Washington University Medical Center between 1997 and 2007. Only cases with surgical pathology material available for review were included in the study. The cases were reviewed by the three study pathologists (RDC, JSL and SEKM) to establish the diagnosis of squamous cell carcinoma and to assess for the presence of nonkeratinizing histology as 

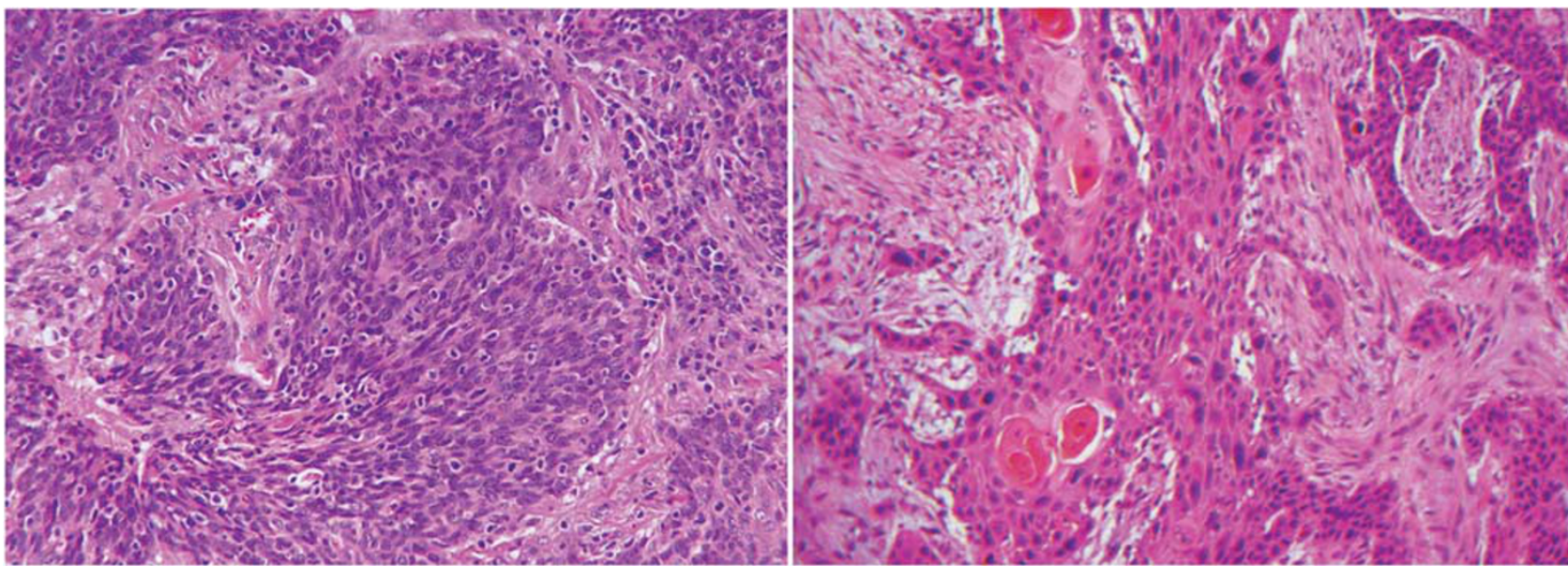

Figure 1 Hematoxylin and eosin-stained sections $(200 \times)$ of nonkeratinizing (left) and keratinizing (right) squamous cell carcinomas. Nonkeratinizing squamous cell carcinoma is composed of large nests that have pushing borders and little stromal reaction. The tumor cells have indistinct cell borders, scant cytoplasm and oval to spindled, hyperchromatic nuclei. Keratinizing squamous cell carcinoma is composed of maturing squamous cells that have polygonal shapes, distinct cell borders and eosinophilic cytoplasm. The tumor nests are infiltrative and there is surrounding stromal desmoplasia.

previously defined. ${ }^{7}$ Examples of nonkeratinizing and keratinizing squamous cell carcinoma morphologic features are shown in Figure 1.

\section{Immunohistochemistry for CDKN2A(p16) and CDKN1A(p21)}

Immunohistochemistry was performed on formalinfixed paraffin-embedded, $4 \mu \mathrm{m}$ tissue sections using an antibody to CDKN2A(p16) (MTM Laboratories, Westborough, MA, USA; mouse monoclonal; 1:1 dilution) or to CDKN1A(p21) (Dako, Carpentaria, CA, USA; mouse monoclonal; 1:25 dilution). Immunostaining was performed on a Ventana Benchmark automated immunostainer (Ventana Medical Systems, Tucson, AZ, USA) according to standard protocols with appropriate positive controls. Antigen retrieval, standard on the machine, utilized the Ventana CC1, EDTA-Tris, pH 8.0 solution. For CDKN2A(p16), cases were classified in a binary manner as positive when $>50 \%$ of cells showed nuclear and cytoplasmic staining or negative when $<50 \%$ of cells stained. For CDKN1A(p21), cases were classified in a binary manner as positive when $>10 \%$ of cells showed nuclear staining or negative when it was $<10 \%$. These cutoff points were based on previously published literature. ${ }^{15,26}$ Slides were interpreted by two study pathologists (RDC and SKEM).

\section{HPV DNA In Situ Hybridization}

DNA in situ hybridization was performed on formalin-fixed, paraffin-embedded, $4 \mu \mathrm{m}$ tissue sections using the ISH I View Blue Plus Detection Kit (Ventana Medical Systems) according to the manufacturer's instructions. The probe hybridizes with the HR HPV genotypes 16, 18, 33, 35, 45, 51, 5256 and 66. Ventana Red Counterstain II (Ventana Medical Systems) was used. Positive staining was identified as blue nuclear dots. Cases were classified in a binary manner as either positive or negative. Any definitive nuclear staining in tumor cells was considered positive. Slides were interpreted by two study pathologists (RDC and SKEM).

\section{HPV PCR}

Tumor-containing areas of paraffin blocks were punched with $1.5 \mathrm{~mm}$ sterile punches. DNA was prepared from these punches using Purgene DNA purification kits (Qiagen). The tumor samples were deparaffinized using a series of xylene and ethanol washes. Next, they were subjected to a rigorous proteinase $\mathrm{K}$ digestion with an incubation time tailored for recovery of DNA. The methodology for DNA purification included RNAse treatment, protein precipitation and DNA precipitation. PCR was then performed using INNO-LiPA HPV Genotyping Extra kits. ${ }^{27}$ Part of the L1 region of the HPV genome was amplified using SPF10 primers. For HR HPV, a 65 -bp fragment was amplified. An additional primer pair for amplification of the human $H L A-D P B 1$ gene was added to monitor sample quality and extraction. For specimens where the HPV amplification product was present on initial reaction, they were hybridized to type-specific probes immobilized as parallel lines on membrane strips pre-made by the manufacturer. After hybridization and stringent washing, streptavidin-conjugated alkaline phosphatase was added. Incubation with BCIP/NBT chromogen yielded a purple precipitate. The results were visually read and compared with the provided interpretation chart to type the HPV.

\section{HPV E6/E7 mRNA RT-PCR}

RT-PCR targeted HPV E6 and E7 RNA from 13 HR HPV types $(16,18,31,33,35,39,45,52,56,58,59$, 
66 and 68). The RT reaction was done with the High Capacity cDNA Reverse Transcription Kit (Applied Biosystems, Carlsbad, CA, USA). All oligo primers in the assays were purchased from Sigma. Each $10 \mu \mathrm{l}$ RT reaction included the following: $1 \times$ RT buffer, $0.4 \mu \mathrm{l}$ of $25 \times \mathrm{dNTP}(100 \mathrm{mM}), 1 \times$ random hexamers, $0.5 \mu \mathrm{l}$ of RT, $0.5 \mu \mathrm{l}$ of RNase inhibitor and $100 \mathrm{ng}$ RNA template. The RT reaction mixture was incubated at $25^{\circ} \mathrm{C}$ for $20 \mathrm{~min}$, then $37^{\circ} \mathrm{C}$ for $60 \mathrm{~min}$ followed by heat-inactivation at $85^{\circ} \mathrm{C}$ for $5 \mathrm{~min}$. Real-time PCR was carried out to quantify the cDNA product using Power SYBR Green PCR Master Mix (Applied Biosystems) and $500 \mathrm{nM} \mathrm{HPV} \mathrm{type-specific}$ primers. Each HPV assay (E6 or E7 from each of the 13 HPV subtypes) was individually performed in a separate well on a 384-well PCR plate. The PCR running protocol was $95^{\circ} \mathrm{C}$ for $10 \mathrm{~min}$, followed by 36 cycles of amplification $\left(95^{\circ} \mathrm{C}\right.$ for $10 \mathrm{~s}, 58^{\circ} \mathrm{C}$ for $15 \mathrm{~s}$ and $60^{\circ} \mathrm{C}$ for $15 \mathrm{~s}$ ). The expression of both GAPDH and $\beta$-actin was used as reference controls for real-time PCR data normalization.

\section{HPV RNA In Situ Hybridization}

In situ hybridization for HR HPV E6/E7 RNA was performed by hand using the RNAscope HPV kit (Advanced Cell Diagnostics, Hayward, CA, USA) according to the manufacturer's instructions as previously described. ${ }^{13}$ Probes targeted the HR HPV genotypes 16, 18, 31, 33, 35, 52 and 58 (performed as a cocktail). Control probes for the bacterial gene $D a p B$ (negative control) and for the housekeeping gene ubiquitin $C$ (positive control) were also included on each case. The slides and controls were read by one study pathologists (RDC) and classified in a binary manner as either positive (brown staining) or negative.

\section{Statistical Analysis}

Fisher exact or $\chi^{2}$ tests were used to examine associations between categorical variables where appropriate. For the continuous variables of age and follow-up length, Student's $t$-tests were performed to evaluate differences by CDKN2A(p16) or CDKN1A(p21) status. Log-rank tests were used to determine differences in overall, disease-free and disease-specific survival by CDKN2A(p16) or CDKN1A(p21) status. Overall survival was calculated from the start date of treatment to the date of death from any cause or the last known follow-up date. Disease-free survival was calculated from the start date of treatment to the date of disease recurrence, or death or last known follow-up if there was no recurrence. Disease-specific survival was calculated from the start date of treatment to the date of death from disease. All statistical tests were twosided, and the level for statistical significance was set at 0.05. SAS 9.1 was used for all major statistical calculations (SAS Institute, Cary, NC, USA).

\section{Results}

\section{Clinicopathologic Features}

A total of 76 cases were identified. The mean length of follow up was 34.6 months (range 2.7-123.7). The mean age was 57.5 years (range 33-81). Fifty-four patients $(71 \%)$ were male and $22(29 \%)$ were female. Fifty-one patients (67\%) were white and twenty-five $(33 \%)$ were African-American. Smoking history was available for all but two patients. The overwhelming majority of patients (71 of 74 or $96 \%$ ) had a history of smoking. Clinical stage was available for all but three patients, and the majority had stage III (21 patients or 29\%) or IV disease (39 patients or $53 \%)$. Nine patients $(12 \%)$ had stage I tumors and four $(6 \%)$ had stage II tumors. Thirty-two patients $(42 \%)$ received definitive radiation-based therapy, whereas forty-four (58\%) received primary surgical treatment followed by radiation. All tumors were clinically laryngeal primary tumors, and only 11 of the $76(15 \%)$ tumors had clinical or pathologic evidence of oropharyngeal extension.

On histologic review of the 76 cases, all but 4 were keratinizing squamous cell carcinomas. The remaining four cases included two basaloid squamous cell carcinomas, one adenosquamous carcinoma and one spindle cell carcinoma. No cases of nonkeratinizing squamous cell carcinoma were identified, and none of the keratinizing tumors contained foci with nonkeratinizing features.

\section{Biomarker Expression}

All cases were evaluated for CDKN2A(p16) and CDKN1A(p21) expression by immunohistochemistry. A significant proportion of cases were positive for either CDKN2A(p16 $\left.{ }^{\mathrm{INK} 4 \mathrm{~A}}\right)$ or CDKN1A(p21) (Table 1). CDKN2A(p16) positivity was seen in 21 cases (28\%) and CDKN1A(p21) positivity was seen in 34 cases $(45 \%)$. Furthermore, there was a strong correlation between CDKN2A(p16) and CDKN1A(p21) positivity with $71 \%$ of CDKN2A(p16)-positive tumors also positive for CDKN1A(p21) compared with $35 \%$ of CDKN2A(p16)-negative tumors $(P=0.0038$, Table 1$)$.

\section{HPV Detection}

Two methods were used to detect HPV DNA; in situ hybridization and PCR for HR genotypes. Two

Table 1 Correlation between CDKN2A(p16) and CDKN1A(p21) expression*

\begin{tabular}{lccr}
$\begin{array}{l}\text { CDKN2A(p16) } \\
\text { status }\end{array}$ & $\begin{array}{c}\text { CDKN1A(p21) } \\
\text { positive }\end{array}$ & $\begin{array}{c}\text { CDKN1A(p21) } \\
\text { negative }\end{array}$ & \multicolumn{1}{c}{ Total } \\
\hline $\begin{array}{l}\text { Positive } \\
\text { Negative }\end{array}$ & $15(20 \%)$ & $6(8 \%)$ & $21(28 \%)$ \\
Total & $19(25 \%)$ & $36(47 \%)$ & $55(72 \%)$ \\
& $34(45 \%)$ & $42(55 \%)$ & $76(100 \%)$
\end{tabular}

${ }^{*} P=0.0038$. 


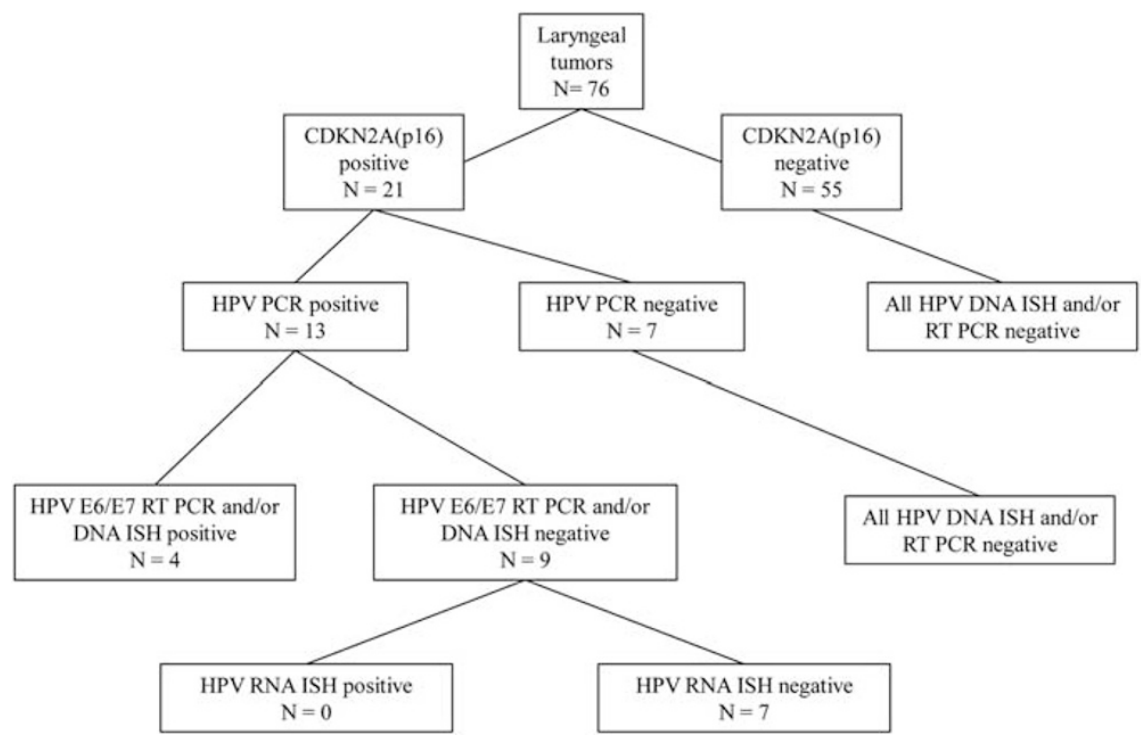

Figure 2 Flow diagram summarizing CDKN2A(p16) and human papillomavirus test results. HPV, human papillomavirus; ISH, in situ hybridization; RT-PCR, reverse transcriptase PCR.

Table 2 HPV DNA ISH and RT-PCR results by CDKN2A(p16) and CDKN1A(p21) status

\begin{tabular}{|c|c|c|c|c|c|}
\hline HPV detection method & All cases & $\begin{array}{l}\text { CDKN2A(p16) } \\
\text { positive, } \mathrm{n}=21\end{array}$ & $\begin{array}{c}C D K N 2 A(p 16) \\
\text { negative, } \mathrm{n}=55\end{array}$ & $\begin{array}{c}C D K N 1 A(p 21) \\
\text { positive, } \mathrm{n}=34\end{array}$ & $\begin{array}{c}C D K N 1 A(p 21) \\
\text { negative, } \mathrm{n}=42\end{array}$ \\
\hline DNA ISH & $3 / 76(4 \%)$ & $3 / 21(14 \%)$ & $0 / 55(0 \%)$ & $3 / 34(9 \%)$ & $0 / 42(0 \%)$ \\
\hline RT-PCR of E6/E7 RNA & $4 / 60(7 \%)$ & $4 / 17(24 \%)$ & $0 / 43(0 \%)$ & $4 / 27(15 \%)$ & $0 / 33(0 \%)$ \\
\hline
\end{tabular}

Abbreviations: HPV, human papillomavirus; ISH, in situ hybridization; RT-PCR, reverse transcriptase PCR.

additional techniques were used to assess for transcriptional activity; RT-PCR and RNA in situ hybridization for HPV E6/E7 mRNA. A flow diagram of the HPV test results is given in Figure 2. DNA in situ hybridization was performed on all cases, and only three (4\%) were positive; all three were also positive for CDKN2A(p16) and CDKN1A(p21). Sufficient material was available for RT-PCR for HPV E6/E7 mRNA in 60 of the 76 cases (18 of the 21 CDKN2A(p16) positive cases had sufficient material for RT-PCR). Four cases were positive (7\%); three for HPV 16 and one for HPV 31. All of these were also positive for CDKN2A(p16) and CDKN1A(p21), and included the three HPV DNA in situ hybridization positive cases. The HPV DNA in situ hybridization and RT-PCR results by CDKN2A(p16) and CDKN1A(p21) status are presented in Table 2.

As a significant proportion of tumors were positive for CDKN2A(p16) and CDKN1A(p21), but only four of these cases were positive for HPV by DNA in situ hybridization or RT-PCR, HPV DNA PCR was performed on just the CDKN2A(p16) positive cases to further evaluate them for HPV, as one would suspect them to be HPV positive given the robustness of $\mathrm{CDKN} 2 \mathrm{~A}(\mathrm{p} 16)$ as a surrogate marker for HPV in the oropharynx. Of the 21
CDKN2A(p16)-positive tumors, 20 had sufficient material for PCR. Thirteen of the twenty (65\%) were in fact HPV positive by DNA PCR. Four cases were HPV type 16, six were HPV 16 plus either HPV 31 or 53, two cases were HPV 31 and one case was HPV 53. All four cases in this subset that were HPV DNA ISH or RT-PCR positive were also positive by PCR, and the HPV types were the same by PCR and RTPCR in these cases. Only one of the HPV DNA PCR positive cases had insufficient material for HPV testing by RT-PCR for HPV E6/ E7 mRNA.

Thus, there were nine cases that were CDKN2A(p16) positive and harbored viral DNA by PCR but were HPV negative by DNA in situ hybridization and/or RT-PCR for HPV E6/E7 mRNA. Because of this discrepancy between the presence of viral DNA and the absence of viral transcripts, another technique was employed to further assess the transcriptional activity in this subgroup; slide-based RNA in situ hybridization was utilized. Eight of the nine cases had sufficient material for RNA in situ hybridization. Of these eight cases, seven were negative for HR HPV RNA, further confirming the lack of transcriptional activity of the virus in these HPV DNA-positive cases. One case was not interpretable due to failure of the positive control. 
Table 3 Clinical characteristics by CDKN2A(p16) and CDKN1A(p21) status

\begin{tabular}{|c|c|c|c|c|c|c|}
\hline \multirow{2}{*}{ Clinical characteristic } & \multicolumn{3}{|c|}{ CDKN2A(p16) status } & \multicolumn{3}{|c|}{ CDKN1A(p21) status } \\
\hline & + & - & $\mathrm{P}$-value & + & - & $\mathrm{P}$-value \\
\hline Age (years, mean \pm s.d.) & $58.8( \pm 11.5)$ & $57.0( \pm 10.8)$ & $P=0.53$ & $59.7( \pm 11.9)$ & $55.7( \pm 9.9)$ & $P=0.11$ \\
\hline $\begin{array}{l}\text { Sex (n) } \\
\text { Male } \\
\text { Female }\end{array}$ & $\begin{array}{r}12 \\
9\end{array}$ & $\begin{array}{l}42 \\
13\end{array}$ & $P=0.098$ & $\begin{array}{l}22 \\
12\end{array}$ & $\begin{array}{l}32 \\
10\end{array}$ & $P=0.27$ \\
\hline $\begin{array}{l}\text { Race (n) } \\
\text { White } \\
\text { African-American }\end{array}$ & $\begin{array}{l}11 \\
10\end{array}$ & $\begin{array}{l}40 \\
15\end{array}$ & $P=0.091$ & $\begin{array}{l}21 \\
13\end{array}$ & $\begin{array}{l}30 \\
12\end{array}$ & $P=0.37$ \\
\hline $\begin{array}{l}\text { Smoking history (n) } \\
\text { Yes } \\
\text { No }\end{array}$ & $\begin{array}{r}19 \\
2\end{array}$ & $\begin{array}{r}52 \\
1\end{array}$ & $P=0.13$ & $\begin{array}{r}32 \\
2\end{array}$ & $\begin{array}{r}39 \\
1\end{array}$ & $P=0.59$ \\
\hline $\begin{array}{l}\text { Tumor stage (n) } \\
\text { I or II } \\
\text { III or IV }\end{array}$ & $\begin{array}{r}4 \\
17\end{array}$ & $\begin{array}{r}9 \\
43\end{array}$ & $P=0.86$ & $\begin{array}{r}7 \\
27\end{array}$ & $\begin{array}{r}6 \\
33\end{array}$ & $P=0.76$ \\
\hline $\begin{array}{l}\text { Treatment strategy (n) } \\
\text { Definitive radiation } \\
\text { Post-operative radiation }\end{array}$ & $\begin{array}{r}13 \\
8\end{array}$ & $\begin{array}{l}19 \\
36\end{array}$ & $P=0.031^{*}$ & $\begin{array}{l}17 \\
17\end{array}$ & $\begin{array}{l}15 \\
27\end{array}$ & $P=0.21$ \\
\hline
\end{tabular}

*Statistically significant, $P<0.05$.

Table 4 Oropharyngeal extension rates by CDKN2A(p16) and CDKN1A(p21) status

\begin{tabular}{|c|c|c|c|c|}
\hline \multirow{2}{*}{$\begin{array}{l}\text { Oropharyngeal } \\
\text { extension }\end{array}$} & \multicolumn{2}{|c|}{$\begin{array}{l}C D K N 2 A(p 16) \\
\text { status }\end{array}$} & \multicolumn{2}{|c|}{$\begin{array}{c}C D K N 1 A(p 21) \\
\text { status }\end{array}$} \\
\hline & + & - & + & - \\
\hline Present & $2(10 \%)$ & $9(16 \%)$ & $4(12 \%)$ & $7(17 \%)$ \\
\hline Absent & $19(90 \%)$ & $46(84 \%)$ & $30(88 \%)$ & $35(83 \%)$ \\
\hline$P$-value & $P=0.45$ & & $P=0.55$ & \\
\hline
\end{tabular}

\section{Clinicopathologic Features and Patient Outcome by CDKN2A(p16) and CDKN1A(p21) Status}

The clinical characteristics of the patients by CDKN2A(p16) and CDKN1A(p21) tumor status are summarized in Table 3. Patients with CDKN2A (p16)-positive tumors were more likely to have received definitive radiation-based therapy than patients with CDKN2A(p16)-negative tumors $(P=0.031)$. There were no other statistically significant differences in age, sex, race, smoking history, tumor stage or treatment strategy by CDKN2A(p16) or CDKN1A(p21) tumor status.

Importantly, neither CDKN2A(p16) nor CDKN1A(p21) status correlated with the presence of oropharyngeal extension (Table 4), making it very unlikely that the positive tumors were oropharyngeal in origin. Of the 11 cases that had oropharyngeal extension, only 2 (18\%) were CDKN2A(p16) positive $(P=0.45)$ and $4(36 \%)$ were CDKN1A(p21) positive $(P=0.55)$. None of the four HPV DNA in situ hybridization or RT-PCR positive cases had oropharyngeal extension.

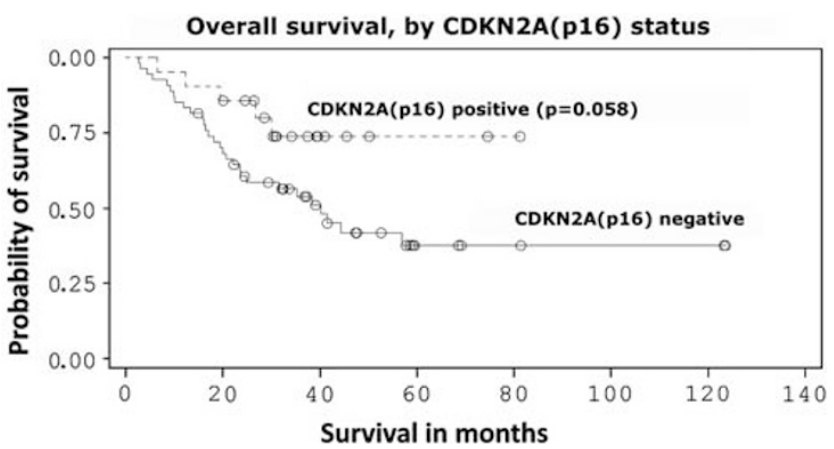

Figure 3 Kaplan-Meier curve of overall survival in patients with CDKN2A(p16) positive versus negative tumors. $P$-values are unadjusted.

Overall survival was better for patients with CDKN1A(p21)-positive tumors than for those with CDKN1A(p21)-negative tumors ( $P=0.045$, Figure 3$)$. There was a trend towards better overall survival for p16-positive tumors as well but this did not reach statistical significance $(P=0.058$, Figure 4$)$. There were no significant differences in disease-free survival by either CDKN2A(p16) or CDKN1A(p21) status. Survival analysis by HPV DNA in situ hybridization or RT-PCR status could not be performed due to the low number of positive cases. However, one of the four patients with an HPV-positive tumor by RT-PCR died with disease within 2 years of diagnosis. The other three were alive and free of disease at last follow-up (minimum follow-up of 2 years).

\section{Discussion}

Few studies have evaluated laryngeal squamous cell carcinomas for the presence of transcriptionally 


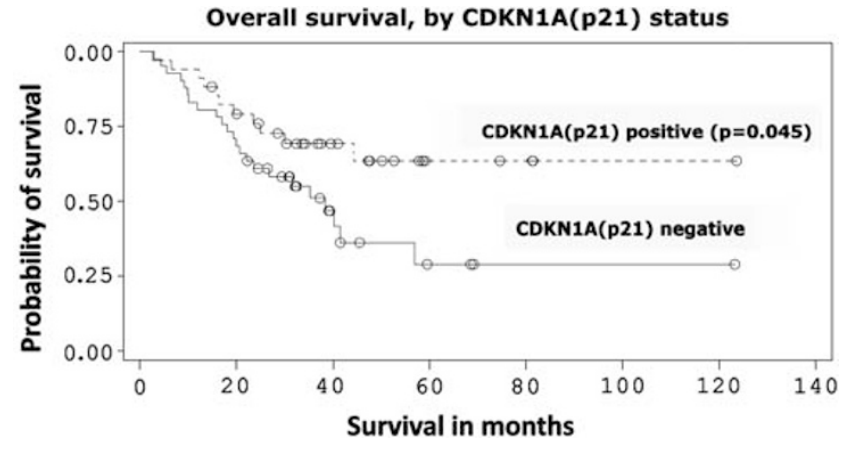

Figure 4 Kaplan-Meier curve of overall survival in patients with CDKN1A(p21) positive versus negative tumors. $P$-values are unadjusted.

active HPV. These have shown a relatively low frequency of virus with small numbers of cases tested. Schlecht et $a l^{24}$ found transcriptionally active HPV in 4 of 27 laryngeal squamous cell carcinomas $(15 \%)$. Although Lewis et $a l^{23}$ found transcriptionally active HPV in 2 of 31 laryngeal squamous cell carcinomas (6.5\%), one of these cases involved the oropharynx and, thus, may have been an oropharynx primary with extensive laryngeal involvement. Our study represents the largest series of laryngeal squamous cell carcinomas evaluated for the presence of transcriptionally active HPV. Four out of sixty cases (7\%) contained HPV E6/E7 mRNA. None of these cases had oropharyngeal extension. HPV DNA in situ hybridization was positive in three of these cases and did not detect any additional HPV-positive cases among the total group of 76 tumors. The low number of tumors with transcriptionally active virus precluded any survival analysis of this group.

As one would expect, all four tumors with transcriptionally active HPV were also CDKN2A (p16) positive. Somewhat surprisingly, however, was the finding of a larger group of CDKN2A(p16) positive and HPV DNA by PCR-positive tumors that were negative for transcriptionally active HPV. A total of 21 of the 76 cases were CDKN2A(p16) positive $(28 \%)$. Thirteen of these were HPV DNA positive by PCR (65\%, one case lacked sufficient material for testing). Of the 13 HPV DNA PCRpositive cases, only 4 were transcriptionally active as shown by RT-PCR.

One could argue that a possible lack of sensitivity of the RT-PCR out of formalin-fixed paraffinembedded tissue may be responsible for the negative RT-PCR results. We therefore used slide-based RNA in situ hybridization for HPV E6/E7 mRNA in the nine HPV DNA PCR positive but not RT-PCRnegative cases. All 7 cases with interpretable results were negative, confirming the absence of transcriptionally active virus.

Thus, it appears that, unlike HPV-related squamous cell carcinoma of the oropharynx, there is not a strong correlation between CDKN2A(p16) expression and transcriptional activation of the virus in squa- mous cell carcinoma of the larynx. The presence of HPV DNA in some tumors may represent a very low viral burden that is incidental 'bystander' virus detectable only by a technique as sensitive as PCR. This seems plausible, as HPV DNA has been found in a significant proportion of benign laryngeal lesions. ${ }^{20}$ Based on these findings, CDKN2A(p16) alone should not be used as a definitive surrogate marker of HPVdriven squamous cell carcinoma in the larynx.

The question remains, what is the significance of CDKN2A(p16) expression in squamous cell carcinoma of the larynx? To date, relatively few other studies have evaluated CDKN2A(p16) expression in laryngeal squamous cell carcinomas. In a relatively small study, Laco et $a l^{22}$ found CDKN2A(p16) overexpression by immunohistochemistry in 58\% of 24 laryngeal squamous cell carcinomas with perfect correlation between CDKN2A(p16) positivity and HPV detected by DNA in situ hybridization. However, the majority of cases showed only focal $(\leq 5 \%)$ immunoreactivity for CDKN2A(p16), which is not the typical pattern seen in oropharyngeal squamous cell carcinoma associated with biologically active HPV, where CDKN2A(p16) expression is strong and diffuse. ${ }^{22}$ Baumann et $a l^{21}$ also reported CDKN2A(p16) overexpression in five of five HPV PCR-positive tumors tested with 'high levels of nuclear and cytoplasmic staining' that was not further quantified. These findings are intriguing, although the number of cases tested is small. In neither of these two studies was transcriptional activation of the virus documented.

We found CDKN2A(p16) overexpression in a significant proportion of tumors $(28 \%)$ using a cutoff for positivity of greater than $50 \%$. This eliminates classification of cases with focal and patchy CDKN2A(p16) immunoreactivity as truly CDKN2A(p16) positive (or overexpressed), as this pattern is also observed in normal squamous mucosa and in a significant minority of non-HPVrelated head and neck squamous cell carcinomas.

Hafkamp et al ${ }^{15}$ recently described CDKN1A(p21) expression as another surrogate marker of HPVrelated tonsillar squamous cell carcinoma. CDKN1A (p21), a tumor-suppressor protein in the TP53 cascade, was also strongly associated with favorable patient outcome. ${ }^{15}$ Fury et al similarly found a correlation between CDKN1A(p21) and CDKN2A (p16) positivity in tonsillar squamous cell carcinoma, although two other studies found no association between HPV and CDKN1A(p21). 16,17,28 Previous studies have also found CDKN1A(p21) overexpression in cervical carcinomas and intraepithelial neoplasia, the majority of which were HPV-related lesions, suggesting that CDKN1A (p21) could have a role in HPV-mediated oncogenesis. ${ }^{29,30}$ In theory, one would expect HPV E6-mediated TP53 inactivation to cause reduced CDKN1A(p21) expression, as CDKN1A(p21) is a downstream mediator of TP53. However, there is some evidence that HPV oncoprotein E7 may be 
responsible for increased CDKN1A(p21) protein levels, as E7 can associate with CDKN1A(p21) and has been shown to increase CDKN1A(p21) expression in HPV-infected genital keratinocytes. ${ }^{31,32}$

Nearly half $(45 \%)$ of the laryngeal squamous cell carcinomas in our study were CDKN1A(p21) positive, a rate comparable to that reported by others. ${ }^{33-35}$ However, previous studies of CDKN1A(p21) in laryngeal carcinomas lack corresponding data on CDKN2A(p16) or HPV status. ${ }^{33-36}$ Ours is the first study to compare CDKN1A(p21) with CDKN2A(p16) and HPV status in laryngeal squamous cell carcinoma. We have found a strong correlation between CDKN1A(p21) and CDKN2A(p16) $(P=0.0038)$, and all four cases with trascriptionally active HPV were not only CDKN2A(p16) positive but also CDKN1A (p21) positive.

Furthermore, CDKN1A(p21) expression was associated with better overall patient survival $(P=0.045)$, whereas CDKN2A(p16) expression showed a trend toward better overall survival, in univariate analysis. There were no significant differences in disease-free survival. Interestingly, a previous study of CDKN1A(p21) in laryngeal squamous cell carcinoma found the opposite-worse survival for patients with CDKN1A(p21)-positive tumors. ${ }^{35}$ Criteria for establishing CDKN1A(p21) positivity may have a role in these divergent results. Jeannon et $a 3^{35}$ considered increased expression of CDKN1A(p21) to be $>50 \%$ positive cells, whereas we used the same criteria of Hafkamp et $a l^{15}$ of $>10 \%$ positive cells. The cutoff point used for establishing CDKN1A(p21) positivity (or overexpression) may be critical as CDKN1A(p21) may have divergent functions depending upon cellular concentration, a concept that warrants further investigation. ${ }^{37}$

In summary, it appears that a subset of laryngeal squamous cell carcinomas display a molecular profile typical of HPV-related squamous cell carcinoma of the oropharynx (CDKN2A(p16) and CDKN1A(p21) biomarker positive). Furthermore, CDKN1A(p21) positivity was associated with better overall patient survival, and there was a trend towards better overall survival for patients with CDKN2A(p16)-positive tumors as well. Improved survival is also a feature of HPV-related oropharyngeal squamous cell carcinoma. However, in contrast to squamous cell carcinoma of the oropharynx, very few of the biomarker positive cases actually harbored transcriptionally active HPV, even though HPV DNA was present in the majority of CDKN2A(p16)-positive laryngeal squamous cell carcinomas. Clearly, the role of HPV in laryngeal squamous cell carcinoma is more complex than in the oropharynx and CDKN2A(p16) and CDKN1A(p21) expression may not reflect HPVdriven tumors in most cases.

\section{Acknowledgements}

We thank Rodney Brown BA, ASCP, Jianping Li BS and Xiaopei Zhu MD for their technical assistance with the immunohistochemistry, ISH and PCR experiments, respectively, and Walter Claremont for his assistance in preparing digital images. We also thank Xiao-Jun Ma PhD, John J Flanagan PhD and Yuling Luo PhD at Advanced Cell Diagnostics for performing the HPV RNA ISH and also acknowledge the support of the Biostatistics Core, Siteman Comprehensive Cancer Center and NCI Cancer Center Support Grant P30 CA091842.

\section{Disclosure/conflict of interest}

The authors declare no conflict of interest.

\section{References}

1 Kreimer AR, Clifford GM, Boyle P, et al. Human papillomavirus types in head and neck squamous cell carcinomas worldwide: a systematic review. Cancer Epidemiol Biomarkers Prev 2005;14:467-475.

2 Syrjanen S. HPV infections and tonsillar carcinoma. J Clin Pathol 2004;57:449-455.

3 Garcia-Milian R, Hernandex H, Panade L, et al. Detection and typing of human papillomavirus DNA in benign and malignant tumours of laryngeal epithelium. Acta Otolaryngol 1998;118:754-758.

4 Duray A, Descamps G, Arafa M, et al. High incidence of high-risk HPV in benign and malignant lesion of the larynx. Int J Oncol 2011;39:51-59.

5 Ang KK, Harris J, Wheeler R, et al. Human papillomavirus and survival of patients wtih oropharyngeal cancer. N Eng J Med 2010;363:24-35.

6 D'Souza G, Kreimer AR, Viscidi R, et al. Case-control study of human papillomavirus and oropharyngeal cancer. N Eng J Med 2007;356:1944-1956.

7 Chernock RD, EL-Mofty SK, Thorstad WL, et al. HPVrelated nonkeratinizing squamous cell carcinoma of the oropharynx: utility of microscopic featurtes in predicting patient outcome. Head Neck Pathol 2009;3: 186-194.

8 El-Mofty SK, Patel S. Human papillomavirus (HPV)related oropharyngeal nonkeratinizing squamous cell carcinoma: characterization of a distinct phenotype. Oral Surg Oral Med Oral Pathol Oral Radiol Endod 2006;101:339-345.

9 Kim SH, Koo BS, Kang S, et al. HPV intergration begins in the tonsillar crypt and leads to the alteration of p16, EGFR and c-myc during tumor formation. Internat J Cancer 2007;120:1418-1425.

10 Boudewijn J, Braakhuis B, Snijders P, et al. Genetic patterns in head and neck cancers that contain or lack transcriptionally active human papillomavirus. J Natl Cancer Inst 2004;96:998-1006.

11 Weinberger $\mathrm{P}$, Yu Z, Haffty B, et al. Molecular classification identifies a subset of human papillomavirus-associated oropharyngeal cancers with favorable prognosis. J Clin Oncol 2006;24:736-747.

12 Klussman JP, Gulitekin E, Weissenborn SJ, et al. Expression of p16 protein identifies a distinct entity of tonsillar carcinomas associated with human papillomavirus. Am J Pathol 2003;162:747-753.

13 Ukpo O, Flanagan J, Ma X, et al. High-risk human papillomavirus E6/E7 mRNA detection by a novel 
in situ hybridization assay strongly correlates wtih p16 expression and patient outcomes in oropharyngeal squamous cell carcinoma. Am J Surg Pathol 2011;35: 1343-1350.

14 Sano T, Oyama T, Kashiwabara K, et al. Expression status of p16 protein is associated with human papillomavirus oncogenic potential in cervical and genital lesions. Am J Pathol 1998;153:1741-1748.

15 Hafkamp HC, Mooren JJ, Claessen SMH, et al. P21Cip1/WAF1 expression is strongly associated with HPV-positive tonsillar carcinoma and a favorable prognosis. Mod Pathol 2009;22:686-698.

16 Chung Y, Lee M, Horng C, et al. Use of combined molecular biomarkers for prediction of clinical outcomes in locally advanced tonsillar cancers treated with chemoradiotherapy alone. Head Neck 2009;31:9-20.

17 Li W, Thompson CH, Cossart YE, et al. The expression of key cell cycle markers and presence of human papillomavirus in squamous cell carcinoma of the tonsil. Head Neck 2004;26:1-9.

18 Abramson A, Steinberg B, Winkler B. Laryngeal papillomatosis: clinical, histopathologic and molecular studies. Laryngoscope 1987;97:678-685.

19 Moore C, Wiatrak B, McClatchey K, et al. High-risk human papillomavirus types and squamous cell carcinoma in patients with respiratory papillomas. Otolaryngol Head Neck Surg 1999;120:698-705.

20 Rihkanen H, Peltomaa J, Syrjanen S. Prevalence of human papillomavirus (HPV) DNA in vocal cords without laryngeal papillomas. Acta Otolaryngol 1994;114:348-351.

21 Baumann JL, Cohen S, Evjen AN, et al. Human papillomavirus in early larygneal carcinoma. Laryngoscope 2009;119:1531-1537.

22 Laco J, Slaninka I, Jirasek M, et al. High-risk human papillomavirus infection and p16INK4a protein expression in laryngeal lesions. Pathol Res Pract 2008;204:545-552.

23 Lewis JS, Ukpo OC, Ma X-J, et al. Transcriptionallyactive high-risk human papillomavirus is rare in oral cavity and laryngeal/hypopharyngeal squamous cell carcinomas-a tissue microarray study utilizing E6/E7 mRNA in situ hybridization. Histopathology 2012;60: 982-991.

24 Schlecht N, Brnadwein-Gensler M, Nuovo G, et al. A comparison of clinically utilized human papillomavirus detection methods in head and neck cancer. Mod Pathol 2011;24:1295-1305.

25 Clayman GL, Stewart MG, Weber RS, et al. Human papillomavirus in laryngeal and hypopharyngeal car- cinomas. Arch Otolaryngol Head Neck Surg 1994;120: 743-748.

26 Ma C, Lewis J Jr. Small biopsy specimens reliably indicate p16 expression status of oropharyngeal squamous cell carcinoma. Head Neck Pathol 2012;6: 208-215.

27 Bello BD, Spinillo A, Alberizzi P, et al. Validation of the SPF10 LiPA human papillomavirus typing assay using formalin-fixed paraffin-embedded cervical biopsy samples. J Clin Microbiol 2009;47:2175-2180.

28 Fury MG, Drobnjak M, Sima CS, et al. Tissue microarray evidence of association between p16 and phosphorylated eIF4E in tonsillar squamous cell carcinoma. Head Neck 2010;33:1340-1345.

29 Milde-Langosch K, Riethdorf S, Kraus-Poppinghaus A, et al. Expression of cyclin-dependent kinase inhibitors p16MTS1, P21WAF1, and p27KIP1 in HPV-positive and HPV-negative cervical adenocarcinomas. Virchows Arch 2001;439:55-61.

30 Skomedal H, Kristensen GB, Lie AK, et al. Aberrant expression of the cell cycle associated proteins TP53, MDM2, p21, p27, cdk4, cyclin D1, RB, and EGFR in cervical carcinomas. Gyn Oncol 1999;73:223-228.

31 Stoppler H, Stoppler MC, Johnson E, et al. The E7 protein of human papillomavirus type 16 sensitizes primary human keratinocytes to apoptosis. Oncogene 1998;17:1207-1214.

32 Funk J, Waga S, Harry J, et al. Inhibition of CDK activity and PCNA-dependent DNA replication by p21 is blocked by interaction with the HPV-16 E7 oncoprotein. Genes Dev 1997;11:2090-2100.

33 Yuen P, Lam K, Choy J, et al. Clinicopathological significance of p53 and p21 expression in the surgical treatment of laryngeal carcinoma. Anticancer Res 2000;20:4863-4866.

34 Pruneri G, Pignataro L, Carboni N, et al. Clinical relevance of expression of the CIP/KIP cell-cycle inhibitors p21 and p27 in laryngeal cancer. J Clin Oncol 1999;17:3150-3159.

35 Jeannon J, Soames J, Lunec J, et al. Expression of cyclin-dependent kinase inhibitor p21(WAF1) and p53 tumour suppressor gene in laryngeal cancer. Clin Otolaryngol 2000;25:23-27.

36 Li X, Izumaru S, Sakamoto K, et al. The immunohistochemical expression of p21WAF1/Cip1 and Proliferating cell nuclear antigen in laryngeal squamous cell carcinomas. J Laryngol Otol 2006;120:1042-1048.

37 Cazzalini O, Scovassi A, Savio M, et al. Multiple roles of the cell cycle inhibitor p21 in the DNA damage response. Mutat Res 2010;704:12-20. 